\title{
Large-signal coherent control of normal modes in quantum-well semiconductor microcavity
}

\author{
Y.-S. Lee ${ }^{\text {a) }}$ and T. B. Norris \\ Center for Ultrafast Optical Science, The University of Michigan, Ann Arbor, Michigan 48109-2099
}

A. Maslov and D. S. Citrin

Department of Physics, Washington State University, Pullman, Washington 99164-2814

J. Prineas, G. Khitrova, and H. M. Gibbs

Optical Sciences Center, University of Arizona, Tucson, Arizona 85721

(Received 1 February 2001; accepted for publication 16 April 2001)

\begin{abstract}
We demonstrate coherent control of the cavity-polariton modes of a quantum-well semiconductor microcavity in a two-color scheme. The cavity enhancement of the excitonic nonlinearity gives rise to a large signal; modulating the relative phase of the excitation pulses between zero and $\pi$ produces a differential reflectivity $(\Delta R / R)$ of up to $20 \%$. The maximum nonlinear signal is obtained for cocircular pump and probe polarization. Excitation-induced dephasing is responsible for the incoherent nonlinear response, and limits the contrast ratio of the optical switching. (C) 2001 American Institute of Physics. [DOI: 10.1063/1.1378316]
\end{abstract}

When a coherent optical pulse is incident on a resonant dipolar absorber, such as the exciton transition in a directgap semiconductor, the coherence of the incident field is transferred to the material polarization. As time elapses, the macroscopic polarization decays due to dephasing, and the population becomes incoherent. If, therefore, before dephasing occurs, a second identical pulse is incident, and the relative phase of the two excitation pulses is $\pi$, then the second pulse coherently de-excites the population. This basic technique of "coherent control" of populations in semiconductors has been demonstrated on the exciton transitions of quantum wells (QWs) and quantum dots. ${ }^{1-5}$

In this letter, we describe the coherent control of the normal modes of a nonperturbatively coupled semiconductor microcavity. This work extends the study of coherent control in several ways. First, the coherent control of a coupled light-matter mode is demonstrated, rather than the coherent control of only a material dipolar response. Second, we investigate coherent control in the nonlinear regime of the excitonic response. Coherent control using strong optical fields is nontrivial since level shifts and dynamic line broadening can occur during the excitation pulses due to many-body effects in the semiconductor. Third, in this work we apply a "two-color" scheme, in which only the lower normal mode (cavity polariton) is excited by the pump pulse pair; the nonlinear response of the system is observed using a probe pulse tuned to the upper normal mode. The probe nonlinearity arises due to the nonlinearity of the exciton transition which underlies both normal modes. The lower mode is pumped in this work because the dephasing time of the lower polariton mode is significantly longer than either the upper mode or the bare exciton. ${ }^{6}$ Fourth, the cavity strongly enhances the optical nonlinearity due to the multiple reflections of the pump and probe fields, which enables very large coherent control signals to be observed. The two-color scheme pro-

${ }^{a)}$ Electronic mail: yunslee@eecs.umich.edu vides a promising tool for the investigation of coherent control in the nonlinear regime since the pump and probe are not degenerate, and may be of interest for all-optical switching. ${ }^{7}$ In this letter we demonstrate the coherent control of the exciton population, and investigate the limitations to the optical switching that arise due to excitation-induced dephasing.

The sample in these experiments (referred to as NMC63) has two $\mathrm{In}_{0.04} \mathrm{Ga}_{0.96} \mathrm{As}$ QWs positioned at the antinodes of a $3 \lambda / 2$ cavity formed by two $99.6 \%$-reflectivity distributed Bragg reflectors [14(16.5) periods of GaAs/AlAs on top (bottom) mirrors]. The QW exciton transition at $1.487 \mathrm{eV}$ (linewidth $0.7 \mathrm{meV}$ ) is resonant with the cavity mode at $10 \mathrm{~K}$, where all experiments were performed. The normal-mode splitting on resonance is $4.5 \mathrm{meV}$. The pump and probe pulses were generated by spectral filtering the output of a 76-MHz mode-locked Ti:sapphire laser producing 75 fs pulses centered at $831 \mathrm{~nm}$. The pump (probe) pulse was resonant with the lower (upper) normal mode, with pulse durations of 190 fs. The spectral filter provided a very high contrast ratio, so that the intensity of the pump (probe) at the upper (lower) mode was less than $10^{-3}$ of its intensity at the lower (upper) mode; hence any effects due to spectral overlap of the pump and probe were completely unobservable. The pump pulse was split into two excitation pulses with variable delay using a Michelson interferometer with a piezoelectric translator in one arm to allow the relative phase between the two pulses to be continuously varied between 0 and $\pi$. The pump and probe beams were overlapped on the sample at near normal incidence; the pump (probe) diameter was $35(20) \mu \mathrm{m}$, and the probe fluence was always less than $10^{-2}$ that of the pump. Probe reflectance spectra were recorded on an optical multichannel analyzer. Photodiodes following a monochromator tuned to the reflectivity minimum of the upper mode were used to record the time-resolved probe differential reflectivity (DR).

Figure 1 shows the probe reflection and DR spectra when the probe delay was set before, between, and after the 


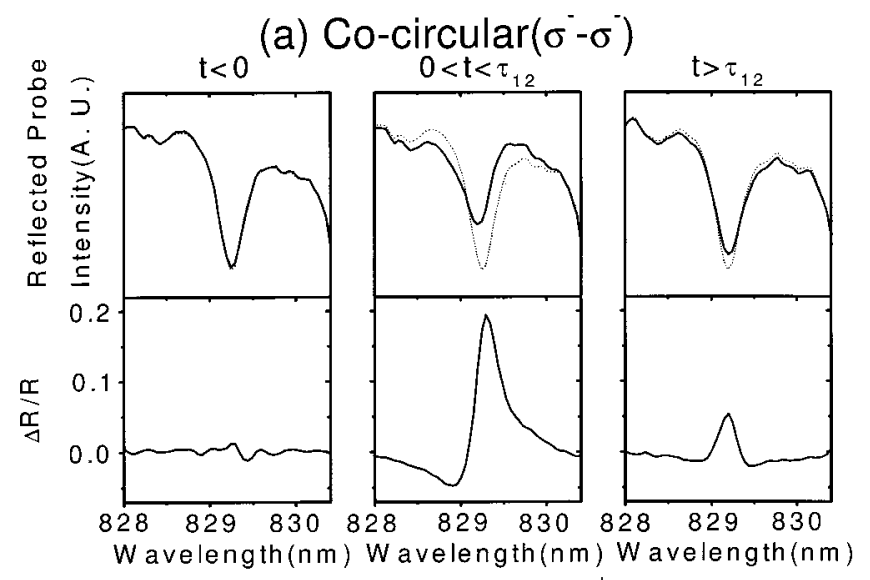

(b) Cross-circular $\left(\sigma^{+}-\sigma\right)$

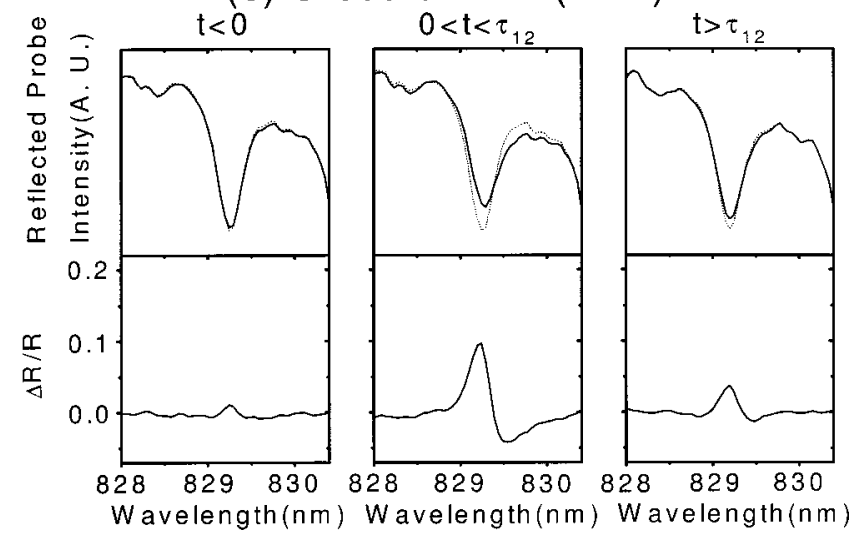

FIG. 1. Temporal evolution of reflection (top) and DR (bottom) spectra for (a) cocircular and (b) cross-circular pump-probe polarization at $t<0$, $0<t<\tau_{12}$, and $t>\tau_{12}$ when single pump pulse fluence is $6.8 \mu \mathrm{J} / \mathrm{cm}^{2}$ pulse and $\tau_{12}$ is $0.9 \mathrm{ps}$. Dotted lines are probe spectra without pump pulses.

two pump pulses for (a) cocircular $\left(\sigma^{-}-\sigma^{-}\right)$and (b) crosscircular $\left(\sigma^{+}-\sigma^{-}\right)$pump and probe polarizations. The pump pulse phase difference was $\pi$. Due to the cavity enhancement, the amplitude of the DR signal $\Delta R / R$ was quite large - up to $20 \%$ - which is at least three orders of magnitude larger than the signal obtained from a bare QW without a cavity. The qualitative form of the DR spectra varies considerably with the pump and probe polarizations. When both pump and probe are cross-circularly polarized, the first pump pulse induces a broadening in the upper mode spectrum, and an increase in the reflectivity at line center. Almost no spectral shift is observed. After the second pulse (delayed from the first pump pulse by $\tau_{12}$ ) the reflectivity spectrum nearly recovers due to the coherent destruction of the exciton population; the line narrows and the reflectivity decreases almost to its $t<0$ value. The recovery is not complete because dephasing between the two pump pulses causes the buildup of an incoherent population which cannot be annihilated by the second pump pulse. When the pump and probe are cocircularly polarized, there is initially a substantial shift of the reflectivity minimum to higher energy. Following the second pump pulse, again there is a substantial recovery of the spectrum. The DR spectrum at long time delay shows no spectral shift, but only a slight broadening.

The coherent control of the exciton population was further demonstrated by fine tuning the temporal delay $\Delta \tau_{12}$ (and hence the phase) between the two pump pulses using the piezoelectric translator in the Michelson with sub-0.01-fs
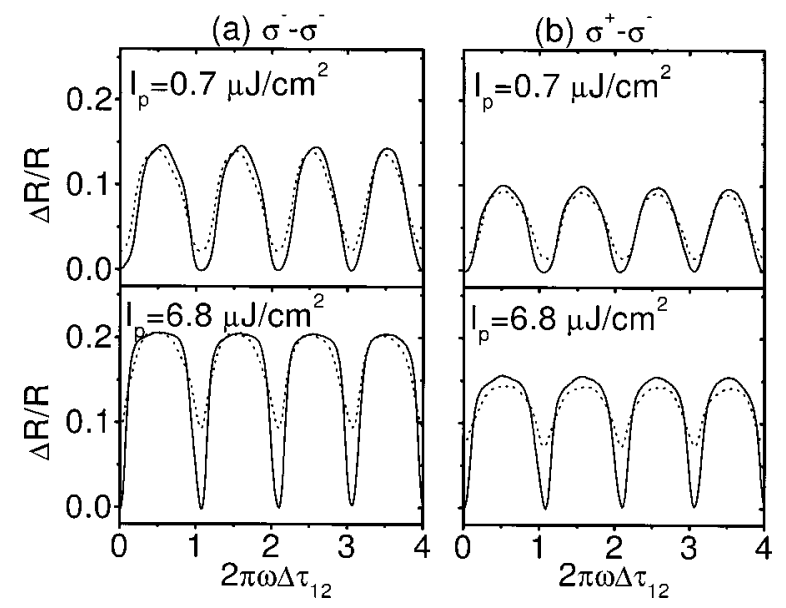

FIG. 2. Interference oscillation of DR at the upper mode resonance wavelength $\left(\lambda=829.25 \mathrm{~nm}\right.$ ) when $\tau_{12}=0.0$ (solid line) and $1.7 \mathrm{ps}$ (dotted line); (a) $\sigma^{-}-\sigma^{-}$; (b) $\sigma^{+}-\sigma^{-}$.

accuracy. The probe delay was fixed at $10 \mathrm{ps}$ following the first pump pulse, so that the DR signal corresponds to the final incoherent population remaining in the system. The oscillations in the population are shown in Fig. 2 for $\tau_{12}=0.0$ and 1.7 ps and pump fluence of 1.4 and $4.1 \mu \mathrm{J} / \mathrm{cm}^{2}$ pulse. The DR signals are measured at the upper mode resonance wavelength $(\lambda=829.25 \mathrm{~nm})$. Several features are to be noted in the data. First, if the system responded linearly to the pump and probe, the fringes would be sinusoidal (as has been observed, for example, in Refs. 1, 2, and 4). This behavior is not observed in our samples even at low fluences. Second, as the pump delay $\tau_{12}$ is changed, the contrast ratio of the fringes decreases due to dephasing between the two pump pulses. Third, the fringe contrast also decreases as the pump fluence increases; this indicates that irreversible excitation induced dephasing (EID) ${ }^{8,9}$ is the limiting factor, particularly for large $\tau_{12}$.

Since the radiative recombination time of a incoherent population of QW excitons ( $>100 \mathrm{ps}$ ) in substantially longer than the dephasing time, $T_{2}(\sim 1 \mathrm{ps})$, the system remains in a quasiequilibrium state long after dephasing has occurred. In order to investigate the optical nonlinearity in the incoherent regime, we measured DR spectra of a probe pulse delayed by 10 ps from a single pump pulse. Figure 3(a) shows the pump-fluence-dependent DR spectra. The peak DR signal depends sublinearly on the pump fluence (figure inset). One might expect in the quasiequilibrium regime that the probe spectra can be modeled using linear dispersion theory; the exciton susceptibility is modeled as

$$
\chi_{\mathrm{QW}}=\frac{\gamma_{R}}{\left(\frac{\lambda_{\mathrm{ex}}}{\lambda}-1\right)+i \gamma}
$$

where $\gamma_{R}$ is the $k_{\|}=0$ radiative decay rate, $\gamma$ the normalized scattering rate, and $\lambda_{\mathrm{ex}}$ the exciton resonance wavelength. Figure 3(b) shows the results of a transfer matrix calculation of the DR spectra where only EID $(\gamma)$ is included; the inset shows the peak DR signal as a function of normalized dephasing rate $\left(\gamma=\gamma_{\mathrm{hom}} / E_{\mathrm{ex}}\right)$. The qualitative agreement with the experimental DR is quite good. As shown in Fig. 1, 


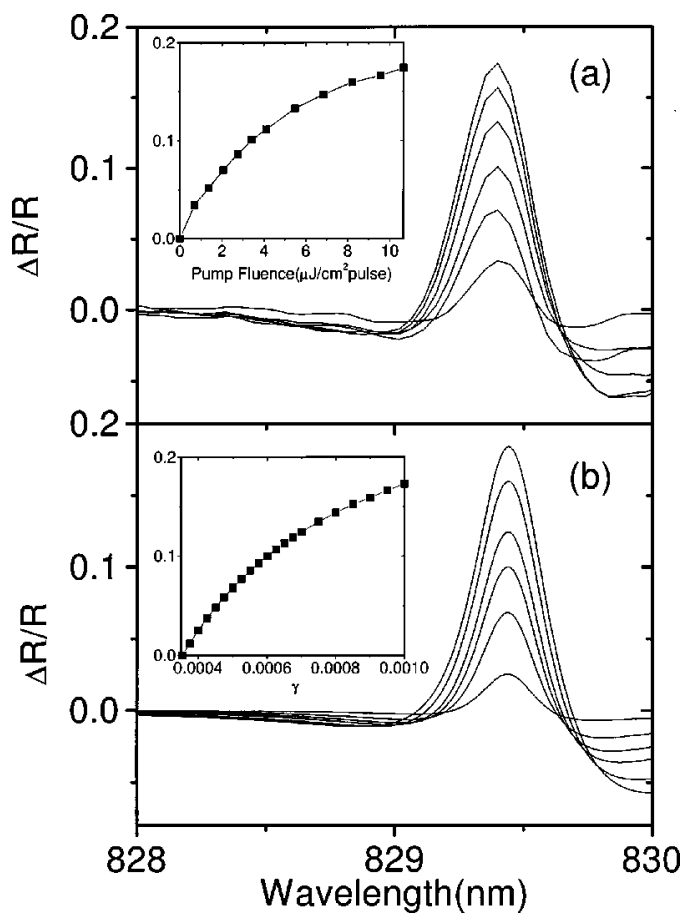

FIG. 3. (a) Pump fluence dependent DR spectra (pump fluence is 0.7, 2.1, $3.4,5.5,8.2$, and $10.9 \mu \mathrm{J} / \mathrm{cm}^{2}$ pulse, and the pump and probe beams are colinearly polarized) and (b) simulation result of scattering rate dependent DR spectra $(\gamma=0.0004,0.0005,0.0006,0.0007,0.0009$, and 0.0011). DR at the peak of the DR spectra vs pump fluence and $\gamma$ is shown in insets.

the EID broadening of the line induced by the first pump is substantially reduced when the population is coherently destroyed by a $\pi$-shifted second pump pulse.

The dynamical behavior of the upper-mode probe response when the lower mode is pumped by a $\pi$-shifted pair of pump pulses is shown in Fig. 4. (The abscissa is the probe delay measured with respect to the first pump pulse.) The DR signals are obtained at the upper mode resonance wavelength $(\lambda=829.25 \mathrm{~nm})$. The dependence on the pulse polarizations is shown in Fig. 4(a); clearly the largest nonlinear response is obtained for cocircular $\left(\sigma^{-}-\sigma^{-}\right)$polarization. The strong polarization dependence, and particularly the enhanced nonlinearity for cocircular polarization arising from the energy shift of the upper mode, is due to exciton-exciton correlations. ${ }^{10-12}$ The energy shift occurs only in the coherent regime between the two pump pulses; by controlling these correlations through the control of the coherent part of the exciton population, large-signal control of the probe nonlinear response is achieved. The degree of coherent control which can be obtained (i.e., the ratio of the maximum to minimum DR signal) is ultimately limited by EID. This is illustrated in the data in Figs. 4(b) and 4(c), which show the effect of pump fluence and delay $\tau_{12}$ on the probe dynamics. Increasing the pump fluence produces a larger peak DR signal, but at the cost of a larger residual incoherent population at long times due to EID. Increasing $\tau_{12}$ also results in a larger residual population due to dephasing.

In summary, we have observed large-signal nondegenerate coherent control of the normal modes in nonperturbatively coupled microcavity. Optimal control is achieved using cocircular polarizations which exploit the strong nonlinearity arising from exciton-exciton correlations in

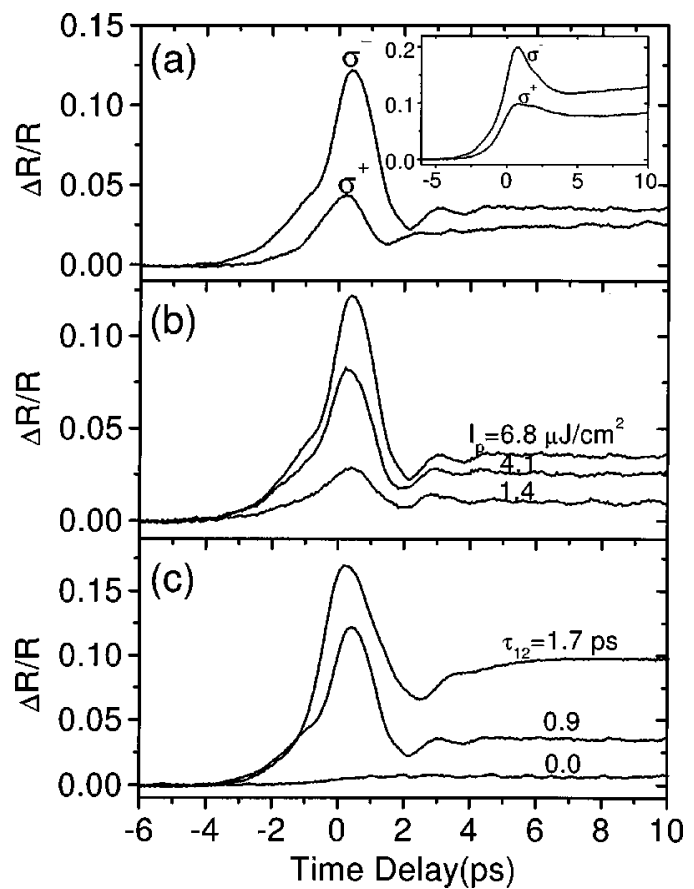

FIG. 4. Time-resolved DR at the upper mode resonance wavelength $(\lambda=829.25 \mathrm{~nm})$ when the phase between two pump pulses is $\pi$. (b) Pump polarization is $\sigma^{+}$, and $\sigma^{-}$probe polarization when pump fluence $\left(I_{p}\right)$ is $6.8 \mu \mathrm{J} / \mathrm{cm}^{2}$ pulse and temporal pump pulse separation $\left(\tau_{12}\right)$ is $0.9 \mathrm{ps}$. Inset: with second pump pulses blocked; (b) pump fluence is 1.4, 4.1, and 6.8 $\mu \mathrm{J} / \mathrm{cm}^{2}$ pulse when $\tau_{12}=0.9 \mathrm{ps}$. (c) Temporal pump pulse separation is 0.0 , $0.9,1.7$ ps when $I_{p}=6.8 \mu \mathrm{J} / \mathrm{cm}^{2}$ pulse. For (b) and(c), pump-probe polarization is $\sigma^{-}-\sigma^{-}$.

QWs. EID governs the nonlinearity at long probe delays, and is the process which ultimately limits the amplitude of the controlled probe signal.

The Michigan portion of the work was supported by AFOSR and by NSF through the Center for Ultrafast Optical Science. The Washington portion was supported by AFOSR, ONR, and NSF. The Arizona portion was supported by DARPA/AFOSR and NSF AMOP.

${ }^{1}$ M. S. C. Luo, S. L. Chuang, P. C. M. Planken, I. Brener, and M. C. Nuss, Phys. Rev. B 48, 11043 (1993).

${ }^{2}$ A. P. Heberle, J. J. Baumberg, K. Köhler, and K. Ploog, Phys. Rev. Lett. 75, 2598 (1995)

${ }^{3}$ J. J. Baumberg, A. P. Heberle, and K. Köhler, J. Opt. Soc. Am. B 13, 1246 (1996).

${ }^{4}$ N. H. Bonadeo, J. Erland, D. Gammon, D. Park, D. S. Katzer, and D. G. Steel, Science 282, 1473 (1998).

${ }^{5}$ X. Marie, P. Le Jeune, T. Amand, M. Brousseau, J. Barrau, M. Paillard, and R. Planel, Phys. Rev. Lett. 79, 3222 (1997).

${ }^{6}$ Y.-S. Lee, T. B. Norris, J. Prineas, G. Khitrova, and H. M. Gibbs, Phys. Status Solidi B 221, 121 (2000).

${ }^{7}$ D. S. Citrin and T. B. Norris, IEEE J. Sel. Top. Quantum Electron. 2, 401 (1996).

${ }^{8}$ H. Wang, K. Ferrio, D. G. Steel, Y. Z. Hu, R. Binder, and S. W. Koch, Phys. Rev. Lett. 71, 1261 (1993).

${ }^{9}$ F. Jahnke, M. Kira, S. W. Kolch, G. Khitrova, E. K. Lindmark, T. R. Nelson, Jr., D. V. Wick, J. D. Berger, O. Lyngnes, H. M. Gibbs, and K. Tai, Phys. Rev. Lett. 77, 5257 (1996).

${ }^{10}$ X. Fan, H. Wang, H. Q. Hou, and B. E. Hammons, Phys. Rev. B 57, R9451 (1998).

${ }^{11}$ Th. Östreich, K. Schönhammer, and L. J. Sham, Phys. Rev. B 58, 12920 (1998).

${ }^{12}$ C. Sieh, T. Meier, F. Jahnke, A. Knorr, S. W. Koch, P. Brick, M. Hübner, C. Ell, J. Prineas, G. Khitrova, and H. M. Gibbs, Phys. Rev. Lett. 82, 3112 (1999). 